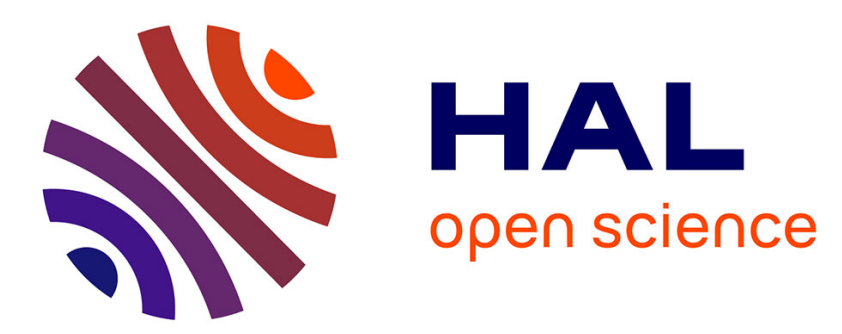

\title{
An adaptive pressure correction method without spurious velocities for diffuse-interface models of incompressible flows
}

\author{
Sebastian Minjeaud
}

\section{- To cite this version:}

Sebastian Minjeaud. An adaptive pressure correction method without spurious velocities for diffuseinterface models of incompressible flows. Journal of Computational Physics, 2013, 236, pp. 143 -156. 10.1016/j.jcp.2012.11.022 . hal-00636296

\section{HAL Id: hal-00636296 \\ https://hal.science/hal-00636296}

Submitted on 27 Oct 2011

HAL is a multi-disciplinary open access archive for the deposit and dissemination of scientific research documents, whether they are published or not. The documents may come from teaching and research institutions in France or abroad, or from public or private research centers.
L'archive ouverte pluridisciplinaire HAL, est destinée au dépôt et à la diffusion de documents scientifiques de niveau recherche, publiés ou non, émanant des établissements d'enseignement et de recherche français ou étrangers, des laboratoires publics ou privés. 


\title{
An adaptive pressure correction method without spurious velocities for diffuse-interface models of incompressible flows
}

\author{
Sebastian Minjeaud \\ Laboratoire J.A. Dieudonné, CNRS UMR 6621, Université de Nice-Sophia Antipolis, France
}

\begin{abstract}
In this article, we propose to study two issues associated with the use of the incremental projection method for solving the incompressible Navier-Stokes equation. The first one is the combination of this time splitting algorithm with an adaptive local refinement method. The second one is the reduction of spurious velocities due to the right-hand side of the momentum balance. We propose a new variant of the incremental projection method for solving the Navier-Stokes equations with variable density and illustrate its behaviour with the example of two phase flows simulations using a Cahn-Hilliard/Navier-Stokes model.
\end{abstract}

Keywords: Incremental projection method, local adaptive refinement, spurious velocities.

\section{Introduction}

Pressure correction schemes [1] are time discretizations of the incompressible Navier-Stokes system. It allows to uncouple the resolution of the momentum balance (convection-diffusion problem) from the divergence free constraint by using a splitting strategy.

In this article, we deal with the incremental projection method [2]. Let us describe this scheme considering first the unsteady Stokes problem:

$$
\begin{cases}\frac{\partial \mathbf{u}}{\partial t}-\Delta \mathbf{u}+\nabla p=\mathbf{f}, & \text { in }] 0, T[\times \Omega, \\ \operatorname{div}(\mathbf{u})=0, & \text { in }] 0, T[\times \Omega,\end{cases}
$$

where $\Omega$ is a smooth connected bounded domain of $\mathbb{R}^{d}(d=2$ or 3$)$ and $T$ is a positive constant. The unknowns are the vector-valued function $\mathbf{u}:] 0, T[\times \Omega \rightarrow$ $\mathbb{R}^{d}$ which stands for the velocity and the scalar function $\left.p:\right] 0, T[\times \Omega \rightarrow \mathbb{R}$ which

Email address: minjeaud@unice.fr (Sebastian Minjeaud) 
stands for the pressure. The vector-valued function $\mathbf{f}:] 0, T\left[\times \Omega \rightarrow \mathbb{R}^{d}\right.$ is a source term which is assumed to be given and smooth. The previous system is supplemented with a Dirichlet boundary condition on the frontier $\Gamma$ of $\Omega$ :

$$
\mathbf{u}=0 \text {, on }] 0, T[\times \Gamma,
$$

and with the following initial condition:

$$
\mathbf{u}(0, \cdot)=0, \quad \text { in } \Omega .
$$

We consider an uniform discretization $0=t^{0}<t^{1}<\cdots<t^{N}=T$ of the time interval ] $0, T\left[\right.$ and we denote by $\Delta t=t^{n+1}-t^{n}(0 \leqslant n \leqslant N-1)$ the time step. To simplify notation, when a function $\mathbf{f}$ is given, we denote by $\mathbf{f}^{n}(0 \leqslant n \leqslant N)$ the value $\mathbf{f}\left(t^{n}, \cdot\right)$ of the function $\mathbf{f}$ at time $t^{n}$.

The projection algorithm is initialized with the prescribed value $\mathbf{u}^{0}=0$ for the velocity and with an arbitrary data $p^{0}$ for the pressure (in practice, we use $\left.p^{0}=0\right)$. Then, assuming that an approximation $\left(\mathbf{u}^{n}, p^{n}\right)$ of the pair velocitypressure at time $t^{n}$ is given, the first step of the projection method consists in computing an intermediate approximation $\widetilde{\mathbf{u}}^{n+1}$ of the velocity at time $t^{n+1}$ forgetting for a moment the divergence free constraint (so that the pressure term can be explicited):

$$
\begin{cases}\frac{\widetilde{\mathbf{u}}^{n+1}-\mathbf{u}^{n}}{\Delta t}-\Delta \widetilde{\mathbf{u}}^{n+1}+\nabla p^{n}=\mathbf{f}^{n+1}, & \text { in } \Omega, \\ \widetilde{\mathbf{u}}^{n+1}=0, & \text { on } \Gamma .\end{cases}
$$

This predicted velocity $\widetilde{\mathbf{u}}^{n+1}$ is then corrected by the resolution of the following problem (of Darcy type), which allows to obtain the approximations $\mathbf{u}^{n+1}$ of the velocity and $p^{n+1}$ of the pressure at time $t^{n+1}$ :

$$
\begin{cases}\frac{\mathbf{u}^{n+1}-\widetilde{\mathbf{u}}^{n+1}}{\Delta t}+\nabla\left(p^{n+1}-p^{n}\right)=0, & \text { in } \Omega \\ \operatorname{div}\left(\mathbf{u}^{n+1}\right)=0, & \text { in } \Omega \\ \mathbf{u}^{n+1} \cdot \mathbf{n}=0, & \text { on } \Gamma\end{cases}
$$

This algorithm is consistent with the continuous problem (1)-(2)-(3) in the sense that when we add the two systems (4) and (5) we obtain:

$$
\frac{\mathbf{u}^{n+1}-\mathbf{u}^{n}}{\Delta t}-\Delta \widetilde{\mathbf{u}}^{n+1}+\nabla p^{n+1}=\mathbf{f}^{n+1}, \text { in } \Omega .
$$

The boundary condition $\mathbf{u}^{n+1} \cdot \mathbf{n}=0$ is reasonable owing to the Dirichlet boundary condition which is imposed to the velocity on $\Gamma$. Moreover, it allows to identify $\mathbf{u}^{n+1}$ as the $\mathrm{L}^{2}(\Omega)$-projection of $\widetilde{\mathbf{u}}^{n+1}$ on the space of divergence free functions with a vanishing normal trace on $\Gamma$ following the Leray decomposition:

$$
\left(\mathrm{L}^{2}(\Omega)\right)^{d}=\left\{\mathbf{v} \in\left(\mathrm{L}^{2}(\Omega)\right)^{d} ; \operatorname{div} \mathbf{v}=0 \text { in } \Omega, \mathbf{v} \cdot \mathbf{n}=0 \text { on } \Gamma\right\} \oplus \nabla\left\{q \in \mathrm{H}^{1}(\Omega)\right\} .
$$


Nevertheless, it is important to remark (this is the main drawback of the incremental projection method) that this boundary condition imposes the following equality for all $n \in \llbracket 0 ; N \rrbracket$ :

$$
\nabla p^{n} \cdot \mathbf{n}=\nabla p^{0} \cdot \mathbf{n}, \text { on } \Gamma .
$$

This pressure boundary condition is artificial (since it is not satisfied by the solution of the continuous problem in general) and leads to a loss of precision [3].

The system (5) (i.e. the projection step) can be solved in two successive sub-steps. Indeed, by taking the divergence of the first equation, the velocity $\mathbf{u}^{n+1}$ is eliminated and we obtain an elliptic equation on the pressure increment $\Phi^{n+1}=p^{n+1}-p^{n}$. Thus, the resolution of system (5) is formally equivalent to:

$$
\left\{\begin{array}{c}
-\Delta \Phi^{n+1}=-\frac{1}{\Delta t} \operatorname{div}\left(\widetilde{\mathbf{u}}^{n+1}\right), \text { in } \Omega, \\
\nabla \Phi^{n+1} \cdot \mathbf{n}=0, \text { on } \Gamma,
\end{array} \text { and } \mathbf{u}^{n+1}=\widetilde{\mathbf{u}}^{n+1}-\Delta t \nabla \Phi^{n+1} \text { in } \Omega .\right.
$$

In practice, the projection method is used in combination with a spatial discretization. We consider in this article a $\mathrm{H}^{1}$-conformal finite element approximation. Let $\mathcal{V}_{h}^{\mathbf{u}}$ and $\mathcal{V}_{h}^{p}$ be two finite element approximation spaces of $\mathcal{V}^{\mathbf{u}}=\mathrm{H}^{1}(\Omega)$ and $\mathcal{V}^{p}=\left\{\nu^{p} \in \mathrm{L}^{2}(\Omega) ; \int_{\Omega} \nu^{p}=0 d x\right\}$ respectively. Since the velocity satisfies an homogeneous Dirichlet boundary condition, we define the following approximation space:

$$
\mathcal{V}_{h, 0}^{\mathbf{u}}=\left\{\nu_{h}^{\mathbf{u}} \in \mathcal{V}_{h}^{\mathbf{u}} ; \nu_{h}^{\mathbf{u}}=0 \text { on } \Gamma\right\} .
$$

We assume that these approximation spaces satisfy the so-called uniform inf-sup condition, i.e. that there exists a positive constant $\beta$ (independent of $h$ ) such that

$$
\inf _{\nu_{h}^{p} \in \mathcal{V}_{h}^{p}} \sup _{\boldsymbol{\nu}_{h}^{\mathbf{u}} \in \mathcal{V}_{h, 0}^{\mathbf{u}}} \frac{\int_{\Omega} \nu_{h}^{p} \operatorname{div} \boldsymbol{\nu}_{h}^{\mathbf{u}} d x}{\left|\nu_{h}^{p}\right|_{\mathrm{L}^{2}(\Omega)}\left|\boldsymbol{\nu}_{h}^{\mathbf{u}}\right|_{\left(\mathrm{H}^{1}(\Omega)\right)^{d}}} \geqslant \beta .
$$

Finally, we assume that the approximation space $\mathcal{V}_{h}^{p}$ is $\mathrm{H}^{1}$-conformal:

$$
\mathcal{V}_{h}^{p} \subset \mathrm{H}^{1}(\Omega) .
$$

In particular, the elliptic problem (7) can naturally be discretized in this space, using the Galerkin method.

Remark 1. These assumptions are for instance satisfied by the Lagrange finite elements $\mathbb{P}_{k+1} / \mathbb{P}_{k}$ for $k \geqslant 1$. We refer to [4] for other examples.

The fully discrete version of the standard incremental projection method can be written as follows: 
Problem 0 (Standard incremental projection method). Let $\mathbf{u}_{h}^{0}=0$ and $p_{h}^{0}=0$. Assume that $\left(\mathbf{u}_{h}^{n}, p_{h}^{n}\right) \in \mathcal{V}_{h, 0}^{\mathbf{u}} \times \mathcal{V}^{p}$ are given $(0 \leqslant n \leqslant N-1)$, then $\left(\mathbf{u}_{h}^{n+1}, p_{h}^{n+1}\right)$ are defined as follows:

- Step 1: Velocity prediction

Find $\widetilde{\mathbf{u}}_{h}^{n+1} \in \mathcal{V}_{h, 0}^{\mathbf{u}}$ such that

$$
\begin{aligned}
\forall \boldsymbol{\nu}_{h}^{\mathbf{u}} \in \mathcal{V}_{h, 0}^{\mathbf{u}}, \quad \int_{\Omega} \frac{\widetilde{\mathbf{u}}_{h}^{n+1}-\mathbf{u}_{h}^{n}}{\Delta t} \cdot \boldsymbol{\nu}_{h}^{\mathbf{u}} d x & +\int_{\Omega} \nabla \widetilde{\mathbf{u}}_{h}^{n+1}: \nabla \boldsymbol{\nu}_{h}^{\mathbf{u}} d x \\
+\int_{\Omega} \boldsymbol{\nu}_{h}^{\mathbf{u}} \cdot \nabla p_{h}^{n} d x & =\int_{\Omega} \mathbf{f}^{n+1} \cdot \boldsymbol{\nu}_{h}^{\mathbf{u}} d x .
\end{aligned}
$$

- Step 2.1: Pressure increment computation

Find $\Phi_{h}^{n+1} \in \mathcal{V}_{h}^{p}$ such that

$$
\forall \nu_{h}^{p} \in \mathcal{V}_{h}^{p}, \quad \int_{\Omega} \nabla \Phi_{h}^{n+1} \cdot \nabla \nu_{h}^{p} d x=\frac{1}{\Delta t} \int_{\Omega} \widetilde{\mathbf{u}}_{h}^{n+1} \cdot \nabla \nu_{h}^{p} d x
$$

- Step 2.2: Pressure correction

Let $p_{h}^{n+1} \in \mathcal{V}_{h}^{p}$ such that

$$
p_{h}^{n+1}=p_{h}^{n}+\Phi_{h}^{n+1} .
$$

- Step 2.3: Velocity correction

Find $\mathbf{u}_{h}^{n+1} \in \mathcal{V}_{h, 0}^{\mathbf{u}}$ such that

$$
\begin{aligned}
\forall \boldsymbol{\nu}_{h}^{\mathbf{u}} \in \mathcal{V}_{h, 0}^{\mathbf{u}}, \quad \int_{\Omega} \mathbf{u}_{h}^{n+1} \cdot \boldsymbol{\nu}_{h}^{\mathbf{u}} d x=\int_{\Omega} \widetilde{\mathbf{u}}_{h}^{n+1} \cdot \boldsymbol{\nu}_{h}^{\mathbf{u}} d x & \\
& \quad-\Delta t \int_{\Omega} \boldsymbol{\nu}_{h}^{\mathbf{u}} \cdot \nabla \Phi_{h}^{n+1} d x
\end{aligned}
$$

The aim of this article is to study the two following issues:

- on one hand, we are interesting in using the incremental projection method in combination with an adaptive local refinement procedure. In this case, the approximation space $\mathcal{V}_{h, 0}^{\mathbf{u}}$ and $\mathcal{V}_{h}^{p}$ are modified at each time step and the pressure correction step has no more sense because it consists in algebraically adding two functions $p_{h}^{n}$ and $\Phi_{h}^{n+1}$ which do not lie in the same approximation spaces. We propose in Section 2 a variant of the projection method and prove that the resulting fully discrete scheme is still stable.

- on the other hand, we are interested in the particular case where the righthand side $\mathbf{f}$ of the momentum balance is a gradient $\mathbf{f}=\nabla Q$ of a function $Q \in \mathrm{H}^{1}(\Omega)$. In this case, the solution of the Stokes equation is trivial: $\mathbf{u}=0$ and $p=Q$ (up to a constant). The incremental projection method is not able to compute exactly this trivial solution and numerical spurious velocities appear during the resolution. In Section 3, we propose a variant of the projection method to overcome this difficulty. 
In Section 4, we illustrate the variants proposed in Sections 2 and 3 by generalizing these schemes to a more intricate situation: the coupling between CahnHilliard and Navier-Stokes equations with variable density for the simulation of two-phase flows.

\section{Conforming adaptive refinement}

The aim of this section is not to describe a particular adaptive refinement procedure but rather to describe the consequences of the use of a local adaptive refinement procedure on the incremental projection method.

When using an adaptive refinement method, the approximation spaces are modified from a time step to the following. This time evolution is described by adding a superscript $n$ or $n+1$ to the notation of approximation spaces in the sequel.

A conseque.texnce of this modification is that the pressure correction step has no more sense. Indeed, since $p_{h}^{n}$ belongs to $\mathcal{V}_{h}^{p, n}$, there is no reason that the sum $p_{h}^{n}+\Phi_{h}^{n+1}$ belongs to the pressure approximation space $\mathcal{V}_{h}^{p, n+1}$ at time $t^{n+1}$. We propose to replace the explicit pressure $p_{h}^{n}$ by its $\mathrm{H}^{1}$-projection on the approximation space $\mathcal{V}_{h}^{p, n+1}$ at time $t^{n+1}$. This leads to the following variant:

Problem 1 (Variant 1). Let $\mathbf{u}_{h}^{0}=0$ and $p_{h}^{0}=0$. Assume that $\left(\mathbf{u}_{h}^{n}, p_{h}^{n}\right) \in$ $\mathcal{V}_{h, 0}^{\mathbf{u}, n} \times \mathcal{V}_{h}^{p, n}$ are given $(0 \leqslant n \leqslant N-1)$, then $\left(\mathbf{u}_{h}^{n+1}, p_{h}^{n+1}\right)$ are defined as follows:

- Step 0: Pressure prediction

Find $\widetilde{p}_{h}^{n+1} \in \mathcal{V}_{h}^{p, n+1}$ such that

$$
\forall \nu_{h}^{p} \in \mathcal{V}_{h}^{p, n+1}, \quad \int_{\Omega} \nabla \widetilde{p}_{h}^{n+1} \cdot \nabla \nu_{h}^{p} d x=\int_{\Omega} \nabla p_{h}^{n} \cdot \nabla \nu_{h}^{p} d x .
$$

- Step 1: Velocity prediction

Find $\widetilde{\mathbf{u}}_{h}^{n+1} \in \mathcal{V}_{h, 0}^{\mathbf{u}, n+1}$ such that

$$
\begin{array}{r}
\forall \boldsymbol{\nu}_{h}^{\mathbf{u}} \in \mathcal{V}_{h, 0}^{\mathbf{u}, n+1}, \quad \int_{\Omega} \frac{\widetilde{\mathbf{u}}_{h}^{n+1}-\mathbf{u}_{h}^{n}}{\Delta t} \cdot \boldsymbol{\nu}_{h}^{\mathbf{u}} d x+\int_{\Omega} \nabla \widetilde{\mathbf{u}}_{h}^{n+1}: \nabla \boldsymbol{\nu}_{h}^{\mathbf{u}} d x \\
-\int_{\Omega} \widetilde{p}_{h}^{n+1} \operatorname{div}\left(\boldsymbol{\nu}_{h}^{\mathbf{u}}\right) d x=\int_{\Omega} \mathbf{f}^{n+1} \cdot \boldsymbol{\nu}_{h}^{\mathbf{u}} d x .
\end{array}
$$

- Step 2.1: Pressure increment computation

Find $\Phi_{h}^{n+1} \in \mathcal{V}_{h}^{p, n+1}$ such that

$$
\forall \nu_{h}^{p} \in \mathcal{V}_{h}^{p, n+1}, \quad \int_{\Omega} \nabla \Phi_{h}^{n+1} \nabla \nu_{h}^{p} d x=\frac{1}{\Delta t} \int_{\Omega} \widetilde{\mathbf{u}}_{h}^{n+1} \cdot \nabla \nu_{h}^{p} d x
$$

- Step 2.2: Pressure correction

Let $p_{h}^{n+1} \in \mathcal{V}_{h}^{p, n+1}$ such that

$$
p_{h}^{n+1}=\widetilde{p}_{h}^{n+1}+\Phi_{h}^{n+1} .
$$




\section{- Step 2.3: Velocity correction}

Find $\mathbf{u}_{h}^{n+1} \in \mathcal{V}_{h, 0}^{\mathbf{u}, n+1}$ such that

$$
\begin{array}{r}
\forall \boldsymbol{\nu}_{h}^{\mathbf{u}} \in \mathcal{V}_{h, 0}^{\mathbf{u}, n+1}, \quad \int_{\Omega} \mathbf{u}_{h}^{n+1} \cdot \boldsymbol{\nu}_{h}^{\mathbf{u}} d x=\int_{\Omega} \\
\widetilde{\mathbf{u}}_{h}^{n+1} \cdot \boldsymbol{\nu}_{h}^{\mathbf{u}} d x \\
+\Delta t \int_{\Omega} \Phi_{h}^{n+1} \operatorname{div} \boldsymbol{\nu}_{h}^{\mathbf{u}} d x .
\end{array}
$$

All steps make sense and performing the projection of $p_{h}^{n}$ in a preliminary step allows to preserve the stability of the method. This result is stated in the following theorem.

Theorem 1. Given $\mathbf{u}_{h}^{n} \in \mathcal{V}_{h, 0}^{\mathbf{u}, n}$ and $p_{h}^{n} \in \mathcal{V}_{h}^{p, n}$, we assume that the triplet $\left(\mathbf{u}_{h}^{n+1}, \widetilde{\mathbf{u}}_{h}^{n+1}, p_{h}^{n+1}\right)$ is a solution of Problem 1. Then, we have the following inequality:

$$
\begin{array}{r}
\frac{1}{2}\left|\mathbf{u}_{h}^{n+1}\right|_{\left(\mathrm{L}^{2}(\Omega)\right)^{d}}^{2}-\frac{1}{2}\left|\mathbf{u}_{h}^{n}\right|_{\left(\mathrm{L}^{2}(\Omega)\right)^{d}}^{2}+\frac{1}{2}\left|\widetilde{\mathbf{u}}_{h}^{n+1}-\mathbf{u}_{h}^{n}\right|_{\left(\mathrm{L}^{2}(\Omega)\right)^{d}}^{2}+\Delta t\left|\nabla \widetilde{\mathbf{u}}_{h}^{n+1}\right|_{\left(\mathrm{L}^{2}(\Omega)\right)^{d}}^{2} \\
+\frac{1}{2}\left[\Delta t^{2}\left|\nabla p_{h}^{n+1}\right|_{\left(\mathrm{L}^{2}(\Omega)\right)^{d}}^{2}-\Delta t^{2}\left|\nabla p_{h}^{n}\right|_{\left(\mathrm{L}^{2}(\Omega)\right)^{d}}^{2}\right] \leqslant \Delta t \int_{\Omega} \mathbf{f}^{n+1} \cdot \widetilde{\mathbf{u}}_{h}^{n+1} d x .
\end{array}
$$

Proof. This proof which is largely inspired from references [5], [6], [7] is split in four steps.

(i) We take $\boldsymbol{\nu}_{h}^{\mathbf{u}}=\Delta t \widetilde{\mathbf{u}}_{h}^{n+1}$ in the system (9). This yields:

$$
\begin{aligned}
& \frac{1}{2}\left|\widetilde{\mathbf{u}}_{h}^{n+1}\right|_{\left(\mathrm{L}^{2}(\Omega)\right)^{d}}^{2}-\frac{1}{2}\left|\mathbf{u}_{h}^{n}\right|_{\left(\mathrm{L}^{2}(\Omega)\right)^{d}}^{2}+\frac{1}{2}\left|\widetilde{\mathbf{u}}_{h}^{n+1}-\mathbf{u}_{h}^{n}\right|_{\left(\mathrm{L}^{2}(\Omega)\right)^{d}}^{2} \\
& +\Delta t\left|\nabla \widetilde{\mathbf{u}}_{h}^{n+1}\right|_{\left(\mathrm{L}^{2}(\Omega)\right)^{d}}^{2}+\Delta t \int_{\Omega} \widetilde{\mathbf{u}}_{h}^{n+1} \cdot \nabla \widetilde{p}_{h}^{n+1} d x=\Delta t \int_{\Omega} \mathbf{f}^{n+1} \cdot \widetilde{\mathbf{u}}_{h}^{n+1} d x .
\end{aligned}
$$

The last term of the left-hand side is not zero since $\widetilde{\mathbf{u}}_{h}^{n+1}$ do not satisfy the divergence free constraint which is actually imposed (in a weak sense) to the function $\widehat{\mathbf{u}}_{h}=\widetilde{\mathbf{u}}_{h}^{n+1}-\Delta t \nabla \Phi_{h}^{n+1}$. Indeed, the pressure increment computation step (10) can be written as follows:

$$
\forall \nu_{h}^{p} \in \mathcal{V}_{h}^{p}, \quad \int_{\Omega} \widehat{\mathbf{u}}_{h} \cdot \nabla \nu_{h}^{p} d x=0 .
$$

(ii) Since $\Phi_{h}^{n+1}=p_{h}^{n+1}-\widetilde{p}_{h}^{n+1}(c f(11))$, we have by definition of $\widehat{\mathbf{u}}_{h}$ :

$$
\widehat{\mathbf{u}}_{h}+\Delta t \nabla p_{h}^{n+1}=\widetilde{\mathbf{u}}_{h}^{n+1}+\Delta t \nabla \widetilde{p}_{h}^{n+1} .
$$

We evaluate the $\mathrm{L}^{2}$ norm of the two sides of this equality. This yields:

$$
\begin{aligned}
& \left|\widehat{\mathbf{u}}_{h}\right|_{\left(\mathrm{L}^{2}(\Omega)\right)^{d}}^{2}+\Delta t^{2}\left|\nabla p_{h}^{n+1}\right|_{\left(\mathrm{L}^{2}(\Omega)\right)^{d}}^{2} \\
& \quad=\left|\widetilde{\mathbf{u}}_{h}^{n+1}\right|_{\left(\mathrm{L}^{2}(\Omega)\right)^{d}}^{2}+\Delta t^{2}\left|\nabla \widetilde{p}_{h}^{n+1}\right|_{\left(\mathrm{L}^{2}(\Omega)\right)^{d}}^{2}+2 \Delta t \int_{\Omega} \widetilde{\mathbf{u}}_{h}^{n+1} \cdot \nabla \widetilde{p}_{h}^{n+1} d x,
\end{aligned}
$$


since the product $2 \Delta t \int_{\Omega} \widehat{\mathbf{u}}_{h} \cdot \nabla p_{h}^{n+1} d x$ vanishes owing to the equality (14).

(iii) Combining (13) and (15) yields:

$$
\begin{gathered}
\frac{1}{2}\left|\widehat{\mathbf{u}}_{h}\right|_{\left(\mathrm{L}^{2}(\Omega)\right)^{d}}^{2}-\frac{1}{2}\left|\mathbf{u}_{h}^{n}\right|_{\left(\mathrm{L}^{2}(\Omega)\right)^{d}}^{2}+\frac{1}{2}\left|\widetilde{\mathbf{u}}_{h}^{n+1}-\mathbf{u}_{h}^{n}\right|_{\left(\mathrm{L}^{2}(\Omega)\right)^{d}}^{2}+\Delta t\left|\nabla \widetilde{\mathbf{u}}_{h}^{n+1}\right|_{\left(\mathrm{L}^{2}(\Omega)\right)^{d}}^{2} \\
+\frac{1}{2} \Delta t^{2}\left[\left|\nabla p_{h}^{n+1}\right|_{\left(\mathrm{L}^{2}(\Omega)\right)^{d}}^{2}-\left|\nabla \widetilde{p}_{h}^{n+1}\right|_{\left(\mathrm{L}^{2}(\Omega)\right)^{d}}^{2}\right]=\Delta t \int_{\Omega} \mathbf{f}^{n+1} \cdot \widetilde{\mathbf{u}}_{h}^{n+1} d x
\end{gathered}
$$

(iv) The last step of the proof consists in remarking that:

- Taking $\nu_{h}^{p}=\widetilde{p}_{h}^{n+1}$ in the pressure prediction step (8) yields:

$$
\left|\nabla \widetilde{p}_{h}^{n+1}\right|_{\left(\mathrm{L}^{2}(\Omega)\right)^{d}} \leqslant\left|\nabla p_{h}^{n}\right|_{\left(\mathrm{L}^{2}(\Omega)\right)^{d}} .
$$

- the velocity correction step (12) defines $\mathbf{u}_{h}^{n+1}$ as the $\mathrm{L}^{2}(\Omega)$ projection of $\widehat{\mathbf{u}}_{h}$ in $\mathcal{V}_{h, 0}^{\mathbf{u}}$. Thus, we have:

$$
\left|\mathbf{u}_{h}^{n+1}\right|_{\left(\mathrm{L}^{2}(\Omega)\right)^{d}} \leqslant\left|\widehat{\mathbf{u}}_{h}\right|_{\left(\mathrm{L}^{2}(\Omega)\right)^{d}} .
$$

These two remarks combined with the inequality (16) yield the conclusion.

\section{Computation of equilibrium states: $\mathrm{f}=\nabla Q$}

We now assume that the right-hand side $\mathbf{f}$ of the momentum balance equation is the gradient of a function $Q \in \mathrm{H}^{1}(\Omega)$.

Since $u^{0}=0$, the exact solution of the Stokes problem (1) is trivial: $\mathbf{u}=0$, $p=Q$ (up to a constant). The issues we are dealing with in this section can be formulated as follows: is the incremental projection method ( $c f$ Problem 0) able to exactly compute this trivial solution when the function $Q$ belongs to the pressure approximation space $\mathcal{V}_{h}^{p}$ ?

Let us begin with a very simple numerical example. This is the 2D simulation of a fluid initially at rest $\left(\mathbf{u}^{0}=0\right)$ confined in the box $\left.\Omega=\right] 0,1\left[^{2}\right.$. The density and the viscosity of the fluid are equal to 1 and the only external force is the gravity $\mathbf{f}=\mathbf{g}$ where $\mathbf{g}=(0,-10)$ is a constant vector. We numerically solve the unsteady Stokes problem using the incremental projection method (cf Problem $0)$.

We use a time step equal to 1 and we use a Taylor-Hood finite element approximation (i.e. a $\mathbb{P}_{2}-\mathbb{P}_{1}$ approximation on triangular meshes and a $\mathbb{Q}_{2}-\mathbb{Q}_{1}$ approximation on quadrangular meshes). The projection method is initialized by choosing $\mathbf{u}^{0}=0$ and $p^{0}=0$. The meshes we use for the computations and the discrete solution $\mathbf{u}_{h}^{1}$ we obtain at the end of the first time step are presented in 
Figure 1 for a structured square $20 \times 20$ mesh (at the right) and for a triangular unstructured mesh (at the left).
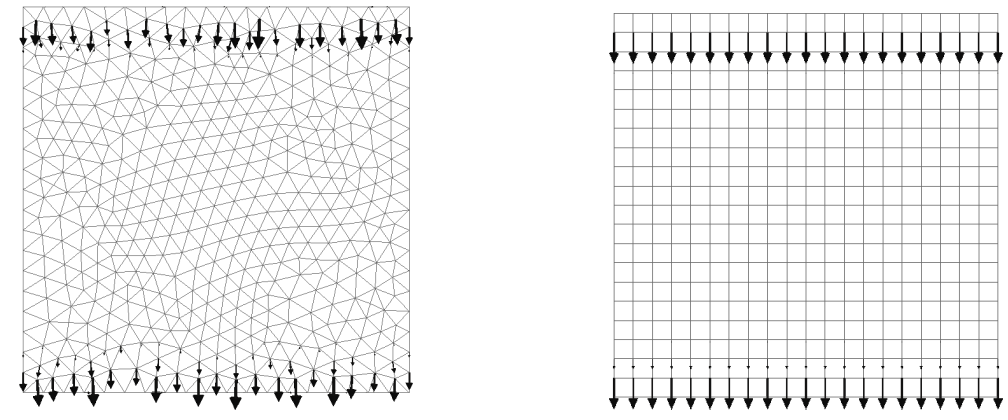

Figure 1: Example of spurious velocities, $\left|\mathbf{u}_{h}^{1}\right|_{L^{\infty}(\Omega)} \sim 10^{-3}$

The exact solution $\left(\mathbf{u} \equiv 0, \quad p=\left(\mathbf{g}_{1}(x-0.5)+\mathbf{g}_{2}(y-0.5)\right)\right)$ of the continuous problem (1) belongs to the discrete approximation space $\mathcal{V}_{h, 0}^{\mathbf{u}} \times \mathcal{V}_{h}^{p}$. However, we observe that the discrete velocity is not zero from the first time step: $\left|\mathbf{u}_{h}^{1}\right|_{\mathrm{L}^{\infty}(\Omega)} \sim 10^{-3}$. The pair $\left(\mathbf{u}_{h}^{n} \equiv 0, p_{h}^{n}=\left(\mathbf{g}_{1}(x-0.5)+\mathbf{g}_{2}(y-0.5)\right)\right)$ for all $n \in \llbracket 1 ; N \rrbracket$, is not a solution of Problem 0 , because of the initialization $\left(\mathbf{u}_{h}^{0} \equiv 0, p_{h}^{0}=0\right)$.

In this simple example, the non zero velocities have small amplitude and are located in the neighborhood of (horizontal) boundaries where the condition $\nabla p_{h}^{n} \cdot \mathbf{n}=0, \forall n \in \llbracket 0 ; N \rrbracket$ imposed by the scheme is not satisfied by the exact solution. But, this phenomenon can have a greater influence if the Navier-Stokes system is coupled to other equations ( $c f$ Section 4$)$.

It would be preferable that the predicted velocity vanishes when the righthand side of the momentum balance is the gradient of a function (possibly depending on the time) which belongs the pressure approximation space $\mathcal{V}_{h}^{p}$.

The idea is to apply the Leray decomposition (6) to the right-hand side $\mathbf{f}$ :

$$
\left\{\begin{array}{l}
\mathbf{f}=\mathbf{u}_{f}+\nabla p_{f} \\
\operatorname{div}\left(\mathbf{u}_{f}\right)=0 \\
\mathbf{u}_{f} \cdot \mathbf{n}=0 \text { on } \Gamma
\end{array}\right.
$$

and to discretize the following system (instead of the system (1)) using the incremental projection method:

$$
\begin{cases}\frac{\partial \mathbf{u}}{\partial t}-\Delta \mathbf{u}+\nabla q=\mathbf{f}-\nabla p_{f}, & \text { in }] 0, T[\times \Omega, \\ \operatorname{div}(\mathbf{u})=0, & \text { in }] 0, T[\times \Omega .\end{cases}
$$

We then set $p=q+p_{f}$. We obtain the following algorithm (a)-(d): 
(a) Velocity prediction:

$$
\frac{\widetilde{\mathbf{u}}^{n+1}-\mathbf{u}^{n}}{\Delta t}-\Delta \widetilde{\mathbf{u}}^{n+1}+\nabla q^{n}=\mathbf{f}^{n+1}-\nabla p_{f}^{n+1}
$$

(b) Pressure increment computation:

$$
-\Delta \Phi^{n+1}=-\frac{1}{\Delta t} \operatorname{div}\left(\widetilde{\mathbf{u}}^{n+1}\right)
$$

(c) Pressure correction: $q^{n+1}=q^{n}+\Phi^{n+1}$.

(d) Velocity correction: $\mathbf{u}^{n+1}=\widetilde{\mathbf{u}}^{n+1}-\Delta t \nabla \Phi^{n+1}$.

We can now go back to the variable $p^{n}=q^{n}+p_{f}^{n}$. Defining $\widetilde{p}^{n+1}=p^{n}+p_{f}^{n+1}-p_{f}^{n}$, the steps $(a)$ and $(c)$ can be written as follows (the steps $(b)$ and $(d)$ remain unchanged):

$\left(a^{\prime}\right)$ Velocity prediction:

$$
\frac{\widetilde{\mathbf{u}}^{n+1}-\mathbf{u}^{n}}{\Delta t}-\Delta \widetilde{\mathbf{u}}^{n+1}+\nabla \widetilde{p}^{n+1}=\mathbf{f}^{n+1} .
$$

$\left(\mathrm{c}^{\prime}\right)$ Pressure correction: $p^{n+1}=\widetilde{p}^{n+1}+\Phi^{n+1}$.

It remains to note that, owing to (17), we can obtain $p_{f}$ by solving:

$$
\begin{cases}-\Delta p_{f}=-\operatorname{div}(\mathbf{f}), & \text { in }] 0, T[\times \Omega, \\ \nabla p_{f} \cdot \mathbf{n}=\mathbf{f} \cdot \mathbf{n}, & \text { on }] 0, T[\times \Gamma .\end{cases}
$$

Consequently, $\widetilde{p}^{n+1}$ is the solution of the following system:

$$
\begin{cases}\Delta \widetilde{p}^{n+1}=\Delta p^{n}+\operatorname{div}\left(\mathbf{f}^{n+1}\right)-\operatorname{div}\left(\mathbf{f}^{n}\right), & \text { in } \Omega, \\ \nabla \widetilde{p}^{n+1} \cdot \mathbf{n}=\nabla p^{n} \cdot \mathbf{n}+\mathbf{f}^{n+1} \cdot \mathbf{n}-\mathbf{f}^{n} \cdot \mathbf{n}, & \text { on } \Gamma .\end{cases}
$$

Thus, we propose the following algorithm:

Problem 2 (Variant 2). Let $\mathbf{u}_{h}^{0}=0$.

- Pressure initialization (only if $n=0$ ): Let $p_{h}^{0}$ be the solution of the following problem:

Find $p_{h}^{0} \in \mathcal{V}_{h}^{p, 0}$ such that

$$
\forall \nu_{h}^{p} \in \mathcal{V}^{p, 0}, \quad \int_{\Omega} \nabla p_{h}^{0} \cdot \nabla \nu_{h}^{p} d x=\int_{\Omega} \mathbf{f}^{0} \cdot \nabla \nu_{h}^{p} d x .
$$


Assume that $\left(\mathbf{u}_{h}^{n}, p_{h}^{n}\right) \in \mathcal{V}_{h, 0}^{\mathbf{u}, n} \times \mathcal{V}^{p, n}$ are given $(0 \leqslant n \leqslant N-1)$, then $\left(\mathbf{u}_{h}^{n+1}, p_{h}^{n+1}\right)$ are defined as follows:

- Step 0: Pressure prediction

Find $\widetilde{p}_{h}^{n+1} \in \mathcal{V}_{h}^{p, n+1}$ such that

$$
\begin{aligned}
\forall \nu_{h}^{p} \in \mathcal{V}^{p, n+1}, \quad \int_{\Omega} \nabla \widetilde{p}_{h}^{n+1} \cdot \nabla \nu_{h}^{p} d x= & \int_{\Omega} \nabla p_{h}^{n} \cdot \nabla \nu_{h}^{p} d x \\
& +\int_{\Omega}\left(\mathbf{f}^{n+1}-\mathbf{f}^{n}\right) \cdot \nabla \nu_{h}^{p} d x .
\end{aligned}
$$

- Step 1: Velocity prediction

Find $\widetilde{\mathbf{u}}_{h}^{n+1} \in \mathcal{V}_{h, 0}^{\mathbf{u}, n+1}$ such that

$$
\begin{array}{r}
\forall \boldsymbol{\nu}_{h}^{\mathbf{u}} \in \mathcal{V}_{h, 0}^{\mathbf{u}, n+1}, \quad \int_{\Omega} \frac{\widetilde{\mathbf{u}}_{h}^{n+1}-\mathbf{u}_{h}^{n}}{\Delta t} \cdot \boldsymbol{\nu}_{h}^{\mathbf{u}} d x+\int_{\Omega} \nabla \widetilde{\mathbf{u}}_{h}^{n+1}: \nabla \boldsymbol{\nu}_{h}^{\mathbf{u}} d x \\
-\int_{\Omega} \widetilde{p}_{h}^{n+1} \operatorname{div}\left(\boldsymbol{\nu}_{h}^{\mathbf{u}}\right) d x=\int_{\Omega} \mathbf{f}^{n+1} \cdot \boldsymbol{\nu}_{h}^{\mathbf{u}} d x .
\end{array}
$$

- Step 2.1: Pressure increment computation

Find $\Phi_{h}^{n+1} \in \mathcal{V}_{h}^{p, n+1}$ such that

$$
\forall \nu_{h}^{p} \in \mathcal{V}_{h}^{p, n+1}, \quad \int_{\Omega} \nabla \Phi_{h}^{n+1} \nabla \nu_{h}^{p} d x=\frac{1}{\Delta t} \int_{\Omega} \widetilde{\mathbf{u}}_{h}^{n+1} \cdot \nabla \nu_{h}^{p} d x .
$$

- Step 2.2: Pressure correction

Let $p_{h}^{n+1} \in \mathcal{V}_{h}^{p, n+1}$ such that

$$
p_{h}^{n+1}=\widetilde{p}_{h}^{n+1}+\Phi_{h}^{n+1} .
$$

- Step 2.3: Velocity correction

Find $\mathbf{u}_{h}^{n+1} \in \mathcal{V}_{h, 0}^{\mathbf{u}, n+1}$ such that

$$
\begin{aligned}
\forall \boldsymbol{\nu}_{h}^{\mathbf{u}} \in \mathcal{V}_{h, 0}^{\mathbf{u}, n+1}, \quad \int_{\Omega} \mathbf{u}_{h}^{n+1} \cdot \boldsymbol{\nu}_{h}^{\mathbf{u}} d x=\int_{\Omega} & \widetilde{\mathbf{u}}_{h}^{n+1} \cdot \boldsymbol{\nu}_{h}^{\mathbf{u}} d x \\
& +\Delta t \int_{\Omega} \Phi_{h}^{n+1} \operatorname{div} \boldsymbol{\nu}_{h}^{\mathbf{u}} d x
\end{aligned}
$$

The advantage of this algorithm is that it allows the computation of the exact solution in the particular case where the right-hand side is the gradient of a function of the pressure approximation space. This is stated in the following proposition:

Proposition 1. Assume that, for all $n \in \llbracket 0 ; N \rrbracket, f^{n}=\nabla q_{h}^{n}$ with $q_{h}^{n} \in \mathcal{V}_{h}^{p}$ and denote by $\left(\mathbf{u}_{h}^{n}, p_{h}^{n}\right)_{n \in \llbracket 0 ; N \rrbracket}$ the approximate solution given by Problem 2. Then

$$
\forall n \in \llbracket 0 ; N \rrbracket, \quad \mathbf{u}_{h}^{n}=0 \quad \text { and } \quad p_{h}^{n}=q_{h}^{n} .
$$


Proof. The proof is made by induction. The initialization step gives $\mathbf{u}_{h}^{0}=0$ and $p_{h}^{0}=q_{h}^{0}$. Let us now assume that, for a given $n$, we have $\mathbf{u}_{h}^{n}=0$ and $p_{h}^{n}=q_{h}^{n}$. Then we obtain after the step 0: $\widetilde{p}_{h}^{n+1}=q_{h}^{n+1}$. The step 1 becomes:

$$
\int_{\Omega} \frac{\widetilde{\mathbf{u}}_{h}^{n+1}}{\Delta t} \cdot \boldsymbol{\nu}_{h}^{\mathbf{u}} d x+\int_{\Omega} \nabla \widetilde{\mathbf{u}}_{h}^{n+1}: \nabla \boldsymbol{\nu}_{h}^{\mathbf{u}} d x=0, \quad \forall \boldsymbol{\nu}_{h}^{\mathbf{u}} \in \mathcal{V}_{h, 0}^{\mathbf{u}} .
$$

This proves that $\widetilde{\mathbf{u}}_{h}^{n+1}=0$, and consequently the step 2.1 gives $\Phi^{n+1}=0$. Finally, the steps 2.2 and 2.3 give $p_{h}^{n+1}=q_{h}^{n+1}$ and $\mathbf{u}_{h}^{n+1}=0$.

Let us go back to the test case (presented at the beginning of this section) of the simulation of a fluid at rest. Proposition 1 states that no spurious velocity are created if we use the Variant 2. This is confirmed by numerical tests: $|\mathbf{u}|_{\mathrm{L}^{\infty}} \sim 10^{-9}$.

Remark 2. - When the right-hand side $\mathbf{f}$ does not depend on the time, the only difference between Variant 2 (cf Problem 2) and the standard algorithm (cf Problem 0) is the pressure initialization step. This is not true when the right-hand side depends on the time.

- The solution of Problem 2 satisfies the following artificial boundary condition:

$$
\forall n \in \llbracket 0 ; N \rrbracket, \quad \nabla p_{h}^{n} \cdot \mathbf{n}=\mathbf{f}^{n} \cdot \mathbf{n} .
$$

- The same idea applied to the non-incremental version (less accurate) of the projection method leads to a similar algorithm; the pressure prediction step is replaced by the following: Find $\widetilde{p}_{h}^{n+1} \in \mathcal{V}_{h}^{p}$ such that

$$
\forall \nu_{h}^{p} \in \mathcal{V}^{p}, \quad \int_{\Omega} \nabla \widetilde{p}_{h}^{n+1} \cdot \nabla \nu_{h}^{p} d x=\int_{\Omega} \mathbf{f}^{n+1} \cdot \nabla \nu_{h}^{p} d x .
$$

Remark 3. - The reasoning done in this section presents some links with the pressure separation algorithm (cf [8] and [9]) for the resolution of Navier-Stokes equations since they consist in subtracting an approximation of the pressure to the two sides of the momentum balance before performing its resolution. This is this idea which led to the equation (18).

- The underlying idea is also very closed from the work of [10] which allows to limit the apparition of spurious velocities. In this reference, the authors use the decomposition (17) and propose to compute in a first step an approximation $q_{h}$ of the "gradient part" $p_{f}$ of the right-hand side $\mathbf{f}$, and then to replace, in the resolution of the Stokes problem, the right hand side $\mathbf{f}$ by $\left(\mathbf{f}-\nabla q_{h}\right)+\nabla\left(\Pi_{h} q_{h}\right)$ where $\Pi_{h}$ is the $\mathrm{L}^{2}$ projection on the pressure approximation space. The computation of $q_{h}$ has to be performed in an approximation space which is larger than the pressure one, the aim being that the two terms $\mathbf{f}-\nabla q_{h}$ and $\nabla\left(\Pi_{h} q_{h}\right)$ generate few spurious velocities: the first because it is close to the "solenoidal part" $\mathbf{u}_{f}$ of the right-hand side and the second because it is the gradient of a function $\left(\Pi_{h} q_{h}\right)$ which 
belongs to the pressure approximation space. This implies that the method used for the resolution of Stokes equations does not generate spurious velocities in the particular case where the right-hand side is the gradient of a function which belongs to the pressure approximation space. Thus, the variant of the projection method proposed above could be used in combination with such methods.

\section{Variable density flows. Coupling with Cahn-Hilliard model}

We give in this section, a more intricate example of the apparition of spurious velocities with two phase flows simulations using a diffuse interface model. In this kind of modelization $[11,12,13,14]$, the interfaces between the two phases are considered as mixing areas with a small but positive thickness $\varepsilon$. The phase $i$ is represented by a smooth phase indicator $c_{i}$ called order parameter (which can be understood here as the volumic fraction of the phase $i$ in the mixture). Thus, the system has as many unknowns as phases. These unknowns vary between 0 and 1 (values which correspond to pure phases by convention) and are linked by the relationship $\sum_{i} c_{i}=1$. Thus, when only two phase are present, the system can be described with a unique order parameter $c=c_{1}=1-c_{2}$.

The two-phase Cahn-Hilliard model is based on the minimization of the following free energy, under the constraint of volume conservation:

$$
\mathcal{F}_{\sigma, \varepsilon}^{\mathrm{diph}}(c)=\int_{\Omega} 12 \frac{\sigma}{\varepsilon} f(c)+\frac{3}{4} \sigma \varepsilon|\nabla c|^{2} d x .
$$

This energy depends on two constant parameters: the surface tension $\sigma$ between the two phases and the interface thickness $\varepsilon$. The function $f$ is called CahnHilliard potential and has a double well structure: $f(c)=c^{2}(1-c)^{2}$.

The Cahn-Hilliard system models the non-miscibility of phase by maintaining the thickness of the mixing area (or interface) at the prescribed value $\varepsilon$. It also allows a volumic representation of capillary forces $\mu \nabla c$ (due to surface tensions between the different phases). The hydrodynamic is taking into account through a coupling with Navier-Stokes equation. The model we consider here is the following:

$$
\left\{\begin{array}{l}
\frac{\partial c}{\partial t}+\mathbf{u} \cdot \nabla c=\operatorname{div}(M(c) \nabla \mu) \\
\mu=\frac{12}{\varepsilon} \sigma f^{\prime}(c)-\frac{3}{2} \sigma \varepsilon \Delta c, \\
\sqrt{\varrho(c)} \frac{\partial}{\partial t}(\sqrt{\varrho(c)} \mathbf{u})+(\varrho(c) \mathbf{u} \cdot \nabla) \mathbf{u}+\frac{\mathbf{u}}{2} \operatorname{div}(\varrho(c) \mathbf{u}) \\
\operatorname{div} \mathbf{u}=0,
\end{array}\right.
$$

The intermediate unknown $\mu$ (which is the functional derivative of the free energy) is called chemical potential. The diffusion coefficient $M$ is called mobility 
and may depend on $c: M(c)=M_{\mathrm{deg}} c^{2}(1-c)^{2}$ where $M_{\mathrm{deg}}$ is a constant coefficient. Note that we adopt a non standard form of the Navier-Stokes equations. Indeed, the density is here a function of the order parameter and does not satisfy the mass conservation equation. Thus, the conservative form or nonconservative form of Navier-Stokes equations do not allow to deduce the kinetic energy balance. The form presented above, initially proposed in [15], enables to obtain the energy balance without using the mass conservation equation. We supplement the previous system with the boundary condition (2) for the velocity, with Neumann homogeneous boundary conditions for the order parameter $c$ and for the chemical potential $\mu$. We assume that the initial condition (3) for the velocity holds and that an initial boundary condition $c(0, \cdot)=c^{0}$ is given for the order parameter $c$.

Let us denote by $\mathcal{V}_{h}^{c}$ and $\mathcal{V}_{h}^{\mu}$ two approximation spaces of $\mathcal{V}^{c}=\mathrm{H}^{1}(\Omega)$ and $\mathcal{V}^{\mu}=\mathrm{H}^{1}(\Omega)$. The standard projection method ( $c f$ Problem 0 ) can be generalized as follows for solving the Cahn-Hilliard/Navier-Stokes model. We denote by $c_{h}^{0} \in \mathcal{V}_{h}^{c}$ an approximation (which is classically obtained by finite element interpolation) of the initial data $c^{0}$.

Problem 3 (Variant 3). Let $\mathbf{u}_{h}^{0}=0$ and $p_{h}^{0}=0$. Assume that $\left(c_{h}^{n}, \mathbf{u}_{h}^{n}, p_{h}^{n}\right) \in$ $\mathcal{V}_{h}^{c, n} \times \mathcal{V}_{h, 0}^{\mathbf{u}, n} \times \mathcal{V}_{h}^{p, n}$ are given.

\section{- Step I: Cahn-Hilliard system}

Find $\left(c_{h}^{n+1}, \mu_{h}^{n+1}\right) \in \mathcal{V}_{h}^{c, n+1} \times \mathcal{V}_{h}^{\mu, n+1}$ such that $\forall \nu_{h}^{c} \in \mathcal{V}_{h}^{c, n+1}, \forall \nu_{h}^{\mu} \in \mathcal{V}_{h}^{\mu, n+1}$,

$$
\left\{\begin{array}{l}
\int_{\Omega} \frac{c_{h}^{n+1}-c_{h}^{n}}{\Delta t} \nu_{h}^{\mu} d x+\int_{\Omega} \nu_{h}^{\mu} \mathbf{u}_{h}^{n} \cdot \nabla c_{h}^{n+1} d x=-\int_{\Omega} M\left(c_{h}^{n}\right) \nabla \mu_{h}^{n+1} \cdot \nabla \nu_{h}^{\mu} d x, \\
\int_{\Omega} \mu_{h}^{n+1} \nu_{h}^{c} d x=\int_{\Omega} \frac{12}{\varepsilon} \sigma f^{\prime}\left(c_{h}^{n+1}\right) \nu_{h}^{c} d x+\int_{\Omega} \frac{3}{2} \sigma \varepsilon \nabla c_{h}^{n+1} \cdot \nabla \nu_{h}^{c} d x,
\end{array}\right.
$$

- Step II.0: Pressure renormalization

Find $\widetilde{p}_{h}^{n+1} \in \mathcal{V}_{h}^{p, n+1}$ such that

$$
\forall \nu_{h}^{p} \in \mathcal{V}_{h}^{p, n+1}, \quad \int_{\Omega} \frac{\nabla \widetilde{p}_{h}^{n+1}}{\sqrt{\varrho_{h}^{n+1}}} \cdot \frac{\nabla \nu_{h}^{p}}{\sqrt{\varrho_{h}^{n+1}}} d x=\int_{\Omega} \frac{\nabla p_{h}^{n}}{\sqrt{\varrho_{h}^{n}}} \cdot \frac{\nabla \nu_{h}^{p}}{\sqrt{\varrho_{h}^{n+1}}} d x .
$$

- Step II.1: Velocity prediction

Find $\widetilde{\mathbf{u}}_{h}^{n+1} \in \mathcal{V}_{h, 0}^{\mathbf{u}, n+1}$ such that, $\forall \boldsymbol{\nu}_{h}^{\mathbf{u}} \in \mathcal{V}_{h, 0}^{\mathbf{u}, n+1}$,

$$
\begin{aligned}
\int_{\Omega} & \sqrt{\varrho^{n+1}} \frac{\sqrt{\varrho^{n+1}} \widetilde{\mathbf{u}}_{h}^{n+1}-\sqrt{\varrho^{n}} \mathbf{u}_{h}^{n}}{\Delta t} \cdot \boldsymbol{\nu}_{h}^{\mathbf{u}} d x \\
& +\frac{1}{2} \int_{\Omega}\left(\varrho^{n+1} \mathbf{u}_{h}^{n} \cdot \nabla\right) \widetilde{\mathbf{u}}_{h}^{n+1} \cdot \boldsymbol{\nu}_{h}^{\mathbf{u}}-\left(\varrho^{n+1} \mathbf{u}_{h}^{n} \cdot \nabla\right) \boldsymbol{\nu} \\
& +\int_{\Omega} 2 \eta^{n+1} D \widetilde{\mathbf{u}}_{h}^{n+1}: D \boldsymbol{\nu}_{h}^{\mathbf{u}} d x-\int_{\Omega} \widetilde{p}_{h}^{n+1} \operatorname{div}\left(\boldsymbol{\nu}_{h}^{\mathbf{u}}\right) d x=\int_{\Omega} \mu_{h}^{n+1} \nabla c_{h}^{n+1} \cdot \boldsymbol{\nu}_{h}^{\mathbf{u}} d x,
\end{aligned}
$$

where $\varrho^{n+1}$ and $\eta^{n+1}$ stand for $\varrho\left(\mathbf{c}_{h}^{n+1}\right)$ and $\eta\left(\mathbf{c}_{h}^{n+1}\right)$. 
- Step II.2.1: Pressure increment computation

Find $\Phi_{h}^{n+1} \in \mathcal{V}_{h}^{p, n+1}$ such that, $\forall \nu_{h}^{p} \in \mathcal{V}_{h}^{p, n+1}$,

$$
\int_{\Omega} \frac{1}{\varrho_{h}^{n+1}} \nabla \Phi_{h}^{n+1} \cdot \nabla \nu_{h}^{p} d x=\frac{1}{\Delta t} \int_{\Omega} \widetilde{\mathbf{u}}_{h}^{n+1} \cdot \nabla \nu_{h}^{p} d x
$$

- Step II.2.2: Pressure correction

Let $p_{h}^{n+1} \in \mathcal{V}_{h}^{p, n+1}$ such that

$$
p_{h}^{n+1}=\widetilde{p}_{h}^{n+1}+\Phi_{h}^{n+1} .
$$

- Step II.2.3: Velocity correction

Find $\mathbf{u}_{h}^{n+1} \in \mathcal{V}_{h, 0}^{\mathbf{u}, n+1}$ such that, $\forall \boldsymbol{\nu}_{h}^{\mathbf{u}} \in \mathcal{V}_{h, 0}^{\mathbf{u}, n+1}$,

$$
\int_{\Omega} \varrho_{h}^{n+1} \mathbf{u}_{h}^{n+1} \cdot \boldsymbol{\nu}_{h}^{\mathbf{u}} d x=\int_{\Omega} \varrho_{h}^{n+1} \widetilde{\mathbf{u}}_{h}^{n+1} \cdot \boldsymbol{\nu}_{h}^{\mathbf{u}} d x+\Delta t \int_{\Omega} \Phi_{h}^{n+1} \operatorname{div} \boldsymbol{\nu}_{h}^{\mathbf{u}} d x .
$$

Remark 4. The pressure renormalization step II.0, initially proposed in [15] enables to ensure the stability of the method in the case where the density is variable.

The previous scheme is also a generalization of Variant 1 ( $c f$ Problem 1) to the case of variable density since the Step II.0 is a projection of the explicit pressure $p^{n}$ on the pressure approximation space $\mathcal{V}_{h}^{p, n+1}$ at time $t^{n+1}$. All steps make sense in the case of local adaptive refinement and the method is stable. However, we are going to show through a simple example that this algorithm can lead to the apparition of spurious velocities and that the work done in Section 3 has to be generalized to variable density flows.

Let us perform the Laplace test case using the scheme presented above: this is the simulation of a $2 \mathrm{D}$ bubble at equilibrium. The test case parameters are given in Table 1.

\begin{tabular}{|c|c|c|c|c|c|c|}
\hline$R$ & $\Omega$ & $\sigma$ & $\varrho_{\mathrm{b}}$ & $\varrho_{\mathrm{l}}$ & $\eta_{\mathrm{b}}$ & $\eta_{\mathrm{l}}$ \\
\hline $10^{-2}$ & {$[0,4 R] \times[0,4 R]$} & 4 & 1 & 1000 & $1.5 \times 10^{-3}$ & $150 \times 10^{-3}$ \\
\hline
\end{tabular}

\begin{tabular}{|c|c|c|}
\hline$\varepsilon$ & $\Delta t$ & $M_{\mathrm{deg}}$ \\
\hline$R / 10$ & $10^{-3}$ & $10^{-6}$ \\
\hline
\end{tabular}

Table 1: Laplace test case parameters

At equilibrium, we may obtain: $\frac{\partial c}{\partial t}=0$ and $\mathbf{u}=0$. Then, the equations imply that $\mu=$ constant and $\nabla p=\mu \nabla c$. Thus, we expect to find:

$$
\left\{\begin{array}{l}
\mathbf{u}=0, \\
p=\mu c(\text { up to a constant), } \\
\mu=\text { constant. }
\end{array}\right.
$$


Furthermore, the Laplace relationship gives the pressure jump expected at the equilibrium:

$$
[p]=\frac{\sigma}{R},
$$

where $R$ is the bubble radius.

The space discretization is performed using multilevel finite element approximation spaces based on a $\mathbb{Q}_{1}$ approximation for the order parameter $c$, the chemical potential $\mu$ and the pressure $p$ and on a $\mathbb{Q}_{2}$ approximation for the velocity $\mathbf{u}$. The reader can refer to [16] for a precise description of the construction of these approximation spaces. The important point is to note that here approximation spaces are not modified during the time marching and that the resolution is accurate in the neighborhood of interface: there is about 10 meshes in the interface.

The initialization of the order parameter is done using an interpolation of the equilibrium profile $c^{\mathrm{eq}}$ of plane interfaces:

$$
c^{0}(x, y)=c^{\mathrm{eq}}\left(\sqrt{x^{2}+y^{2}}-R\right)=\frac{1}{2}-\frac{1}{2} \tanh \left(\frac{2\left(\sqrt{x^{2}+y^{2}}-R\right)}{\varepsilon}\right) .
$$

The results we obtain at different time steps are presented in Figure 2. The solid lines represent 10 contour levels (between 0.1 and 0.9 ) of the order parameter $c$ and the dotted lines represent 15 contour levels of the stream function. We observe that spurious velocities appear in the neighborhood of the interface which becomes unstable after few time steps.

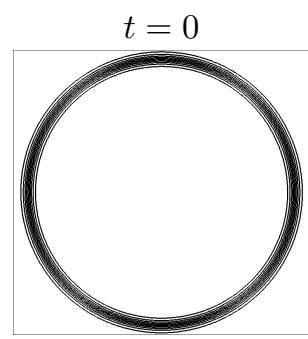

$\mathrm{KE}=0$

$\|\mathbf{u}\|_{\infty}=0$

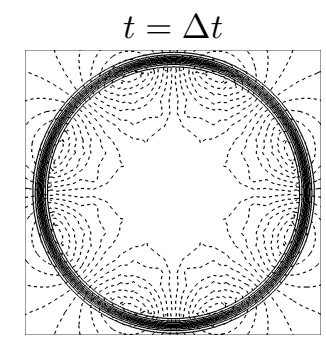

$\mathrm{KE}=8.17 \times 10^{-7}$

$\|\mathbf{u}\|_{\infty}=0.23$

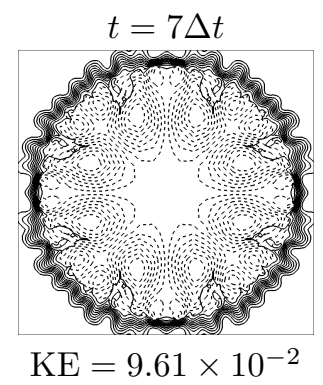

$\|\mathbf{u}\|_{\infty}=32.9$

Figure 2: Contour level of the order parameter (solid lines), streamlines (dotted lines), kinetic energy (KE) and max norm of the velocity.

This phenomenon can be explained by the following two reasons:

- At the initialization, the order parameter profile we chose does not allow to obtain a constant chemical potential $\mu$. Consequently, the right-hand side of the momentum balance is not a gradient. There is no chance to obtain a solution with a vanishing velocity. 
- The projection method presented in Problem 3 does not allow to compute the solutions $\mathbf{u}_{h}=0$ and $p_{h}=q_{h}$ of the Navier-Stokes equation in the particular case where the right-hand side is a gradient $\nabla q_{h}, q_{h} \in \mathcal{V}_{h}^{p}$.

Concerning the initialization problem, it is difficult to bring a solution since the analytical expression of the equilibrium profile is known only for plane interfaces on an infinite domain. We propose to numerically compute the equilibrium profile.

Assume that the equilibrium profile is reached and that the order parameter is constant far from interfaces. Let us denote by $c_{0}$ and $c_{\infty}$ the constant values of order parameters in each phase. Owing to equations (19) and (20), the CahnHilliard system gives:

$$
f^{\prime}\left(c_{0}\right)=f^{\prime}\left(c_{\infty}\right)=\frac{\varepsilon}{12 R}\left(c_{0}-c_{\infty}\right) .
$$

In practice we numerically solved the polynomial equations $f^{\prime}\left(c_{0}\right)=f^{\prime}\left(c_{\infty}\right)=$ $\frac{\varepsilon}{12 R}$ and use the values we obtain to initialize order parameters:

$$
c^{0}(x, y)=\left(c_{0}-c_{\infty}\right) c^{\mathrm{eq}}(x, y)+c_{\infty} .
$$

Then, we perform (before the beginning of the computation) few time iterations solving only the Cahn-Hilliard system with a constant mobility $M \equiv 1$ to obtain a numerical solution which is very closed from the steady solution. We manage to obtain a discrete solution such that $\left|\mu_{\max }-\mu_{\min }\right| \sim \mid$ machine epsilon| for all meshes.

Concerning the projection method, owing to Section 3, we propose the following variant:

\section{Problem 4 (Variant 4). - Step I.: Cahn-Hilliard system resolution}

This step remains unchanged (cf Problem 3).

- Initialization (only if $n=0$ ): Let $\mathbf{u}_{h}^{0}=0$ and $p_{h}^{0}$ the solution of the following problem:

Find $p_{h}^{0} \in \mathcal{V}_{h}^{p, 0}$ such that

$$
\forall \nu_{h}^{p} \in \mathcal{V}^{p, 0}, \quad \int_{\Omega} \frac{1}{\sqrt{\varrho^{0}} \sqrt{\varrho^{1}}} \nabla p_{h}^{0} \cdot \nabla \nu_{h}^{p} d x=\int_{\Omega} \frac{1}{\sqrt{\varrho^{0}} \sqrt{\varrho^{1}}} \mathbf{f}^{0} \cdot \nabla \nu_{h}^{p} d x .
$$

Assume that $\left(\mathbf{u}_{h}^{n}, p_{h}^{n}\right) \in \mathcal{V}_{h, 0}^{\mathbf{u}, n} \times \mathcal{V}^{p, n}$ are given $(0 \leqslant n \leqslant N-1),\left(\mathbf{u}_{h}^{n+1}, p_{h}^{n+1}\right)$ are defined as follows:

- Step II.0: Pressure prediction

Find $\widetilde{p}_{h}^{n+1} \in \mathcal{V}_{h}^{p, n+1}$ such that, $\forall \nu_{h}^{p} \in \mathcal{V}_{h}^{p, n+1}$,

$$
\begin{aligned}
\int_{\Omega} \frac{1}{\varrho^{n+1}} \nabla \widetilde{p}_{h}^{n+1} \nabla \nu_{h}^{p} d x & =\int_{\Omega} \frac{1}{\sqrt{\varrho^{n}} \sqrt{\varrho^{n+1}}} \nabla p_{h}^{n} \nabla \nu_{h}^{p} d x \\
& +\int_{\Omega}\left(\frac{\mathbf{f}^{n+1}}{\sqrt{\varrho^{n+1}}}-\frac{\mathbf{f}^{n}}{\sqrt{\varrho^{n}}}\right) \cdot \frac{\nabla \nu_{h}^{p}}{\sqrt{\varrho^{n+1}}} d x,
\end{aligned}
$$


where $\mathbf{f}^{n}=\mu_{h}^{n} \nabla c_{h}^{n}+\varrho\left(c_{h}^{n+1}\right) \mathbf{g}$.

- Step II.1: Velocity prediction

Find $\widetilde{\mathbf{u}}_{h}^{n+1} \in \mathcal{V}_{h, 0}^{\mathbf{u}, n+1}$ such that, $\forall \boldsymbol{\nu}_{h}^{\mathbf{u}} \in \mathcal{V}_{h, 0}^{\mathbf{u}, n+1}$,

$$
\begin{aligned}
& \int_{\Omega} \sqrt{\varrho^{n+1}} \frac{\sqrt{\varrho^{n+1}} \widetilde{\mathbf{u}}_{h}^{n+1}-\sqrt{\varrho^{n}} \mathbf{u}_{h}^{n}}{\Delta t} \cdot \boldsymbol{\nu}_{h}^{\mathbf{u}} d x \\
& \quad+\frac{1}{2} \int_{\Omega}\left(\varrho^{n+1} \mathbf{u}_{h}^{n} \cdot \nabla\right) \widetilde{\mathbf{u}}_{h}^{n+1} \cdot \boldsymbol{\nu}_{h}^{\mathbf{u}}-\left(\varrho^{n+1} \mathbf{u}_{h}^{n} \cdot \nabla\right) \boldsymbol{\nu}_{h}^{\mathbf{u}} \cdot \widetilde{\mathbf{u}}_{h}^{n+1} d x \\
& \quad+\int_{\Omega} 2 \eta^{n+1} D \widetilde{\mathbf{u}}_{h}^{n+1}: D \boldsymbol{\nu}_{h}^{\mathbf{u}} d x-\int_{\Omega} \widetilde{p}_{h}^{n+1} \operatorname{div}\left(\boldsymbol{\nu}_{h}^{\mathbf{u}}\right) d x=\int_{\Omega} \mathbf{f}^{n+1} \cdot \boldsymbol{\nu}_{h}^{\mathbf{u}} d x,
\end{aligned}
$$

where $\varrho^{n+1}$ and $\eta^{n+1}$ stands for $\varrho\left(c_{h}^{n+1}\right)$ and $\eta\left(c_{h}^{n+1}\right)$ respectively.

- Step II.2.1: Pressure increment computation

Find $\Phi_{h}^{n+1} \in \mathcal{V}_{h}^{p, n+1}$ such that

$$
\forall \nu_{h}^{p} \in \mathcal{V}_{h}^{p, n+1}, \quad \int_{\Omega} \frac{1}{\varrho_{h}^{n+1}} \nabla \Phi_{h}^{n+1} \nabla \nu_{h}^{p} d x=\frac{1}{\Delta t} \int_{\Omega} \widetilde{\mathbf{u}}_{h}^{n+1} \cdot \nabla \nu_{h}^{p} d x .
$$

- Step II.2.2: Pressure correction

Let $p_{h}^{n+1} \in \mathcal{V}_{h}^{p, n+1}$ such that

$$
p_{h}^{n+1}=\widetilde{p}_{h}^{n+1}+\Phi_{h}^{n+1} .
$$

- Step II.2.3: Velocity correction

Find $\mathbf{u}_{h}^{n+1} \in \mathcal{V}_{h, 0}^{\mathbf{u}, n+1}$ such that

$$
\begin{aligned}
\forall \boldsymbol{\nu}_{h}^{\mathbf{u}} \in \mathcal{V}_{h, 0}^{\mathbf{u}, n+1}, \quad \int_{\Omega} \varrho_{h}^{n+1} \mathbf{u}_{h}^{n+1} \cdot \boldsymbol{\nu}_{h}^{\mathbf{u}} d x=\int_{\Omega} \varrho_{h}^{n+1} \widetilde{\mathbf{u}}_{h}^{n+1} \cdot \boldsymbol{\nu}_{h}^{\mathbf{u}} d x \\
\quad+\Delta t \int_{\Omega} \Phi_{h}^{n+1} \operatorname{div} \boldsymbol{\nu}_{h}^{\mathbf{u}} d x
\end{aligned}
$$

The advantage of this method is the same as in Section 3. Assume that $\mathbf{f}^{n}=\nabla q_{h}^{n}, \quad \forall n \in \mathbb{N}$, and that $\mathbf{u}_{h}^{n}=0$ and $p_{h}^{n}=q_{h}^{n}$ then we obtain after the step II.0: $\widetilde{p}_{h}^{n+1}=q_{h}^{n+1}$. Thus, the step II.1 becomes:

$$
\int_{\Omega} \varrho^{n+1} \frac{\widetilde{\mathbf{u}}_{h}^{n+1}}{\Delta t} \cdot \mathbf{v}_{h} d x+\int_{\Omega} 2 \eta^{n+1} D \widetilde{\mathbf{u}}_{h}^{n+1}: D \mathbf{v}_{h} d x=0, \quad \forall \mathbf{v}_{h} \in \mathcal{V}_{h, 0}^{\mathbf{u}} .
$$

The solution is $\widetilde{\mathbf{u}}_{h}^{n+1}=0$. Then the step II.2.1 implies that $\Phi^{n+1}=0$ and the steps II.2.2 and II.2.3 give $p_{h}^{n+1}=q_{h}^{n+1}$ and $\mathbf{u}_{h}^{n+1}=0$.

Let us go back to the Laplace test case. The results we obtain using this variant of the incremental projection method are presented in Figure 3. On this academic test case, the spurious velocities are completely eliminated. 


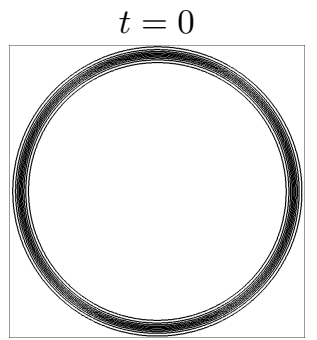

$\mathrm{KE}=0$

$\|\mathbf{u}\|_{\infty}=0$

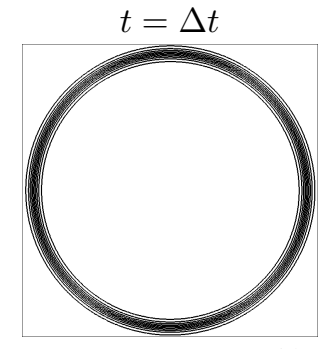

$\mathrm{KE}=2.0 \times 10^{-31}$

$\|\mathbf{u}\|_{\infty}=2.48 \times 10^{-14}$

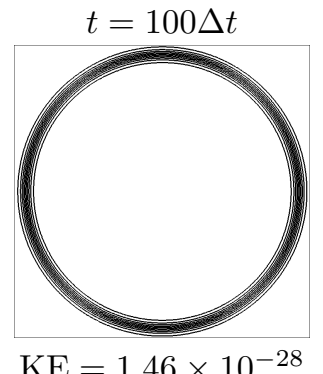

$\mathrm{KE}=1.46 \times 10^{-28}$

$\|\mathbf{u}\|_{\infty}=5.7 \times 10^{-14}$

Figure 3: Variant 2: contour level of the order parameter, kinetic energy (KE) and max norm of the velocity.

To conclude this section, we provide a numerical simulation of a $2 \mathrm{D}$ gas bubble rising in a liquid column.

The space discretization is performed on square local adaptive refined meshes using:

- $\mathbb{Q}_{1}$ Lagrange finite element for the order parameter $c$, the chemical potential $\mu$ and for the pressure $p$,

- $\mathbb{Q}_{2}$ Lagrange finite element for each component of the velocity $\mathbf{u}$.

The adaptation procedures are based on conforming multilevel finite element approximation spaces which are built by refinement or unrefinement of the finite element basis functions instead of cells. All the details about this method and also various examples (in particular, simulations using the Cahn-Hilliard model considered in this article) are described in [16]. The refinement criterion used in those (un-)refinement procedures imposes the value of the smaller diameter $h_{\text {min }}$ of a cell and ensures that refined areas are located in the neighborhood of the interfaces (i.e. where no order parameter is equal to one). We do not give more details on spatial discretization issues here since the main goal of this article is to investigate the properties of time discretization scheme.

The test case parameters are given in Table 2. This corresponds to a Bond number equal to 1 and a Morton number equal $5 \times 10^{-3}$. In this regime, a bubble keeps its spherical shape during its rise [17, 18]. We observe the same kind of behaviour here even if we perform $2 \mathrm{D}$ simulations. 


\begin{tabular}{|c|c|c|c|c|}
\hline$R$ & $\Omega$ & $\sigma$ & $\varrho_{\mathrm{b}}$ & $\varrho_{\mathrm{l}}$ \\
\hline $1.336 \times 10^{-3}$ & {$[-4 R, 4 R] \times[0,24 R]$} & 0.07 & 1 & 1000 \\
\hline
\end{tabular}

\begin{tabular}{|c|c|}
\hline$\eta_{\mathrm{b}}$ & $\eta_{\mathrm{l}}$ \\
\hline $1.15 \times 10^{-3}$ & $1.15 \times 10^{-1}$ \\
\hline
\end{tabular}

\begin{tabular}{|c|c|c|c|c|}
\hline$\varepsilon$ & $h_{\min }$ & $\varepsilon / h_{\min }$ & $\Delta t$ & $M_{\mathrm{deg}}$ \\
\hline$R / 10$ & $R / 10$ & 1 & $10^{-4}$ or $5 \times 10^{-4}$ & $10^{-5}$ \\
\hline
\end{tabular}

Table 2: Rising bubble. Test case parameters

We perform the simulation for time steps $\Delta t$ equal to $10^{-4}$ and $5 \times 10^{-4}$ using the Variants 3 and 4 of the incremental projection method ( $c f$ Problems 3 and 4). The time evolution of the system is presented in Figure 4. We observe that the volume of the bubble is not conserved when using the Variant 3. A more precise representation of the time evolution of the bubble volume is given in Figure 5 using both the Variants 3 and 4 for $\Delta t=10^{-4}$ and $\Delta t=5 \times 10^{-4}$. The represented quantity is computed by adding the area of all cells $K$ such that $\int_{K} c_{h}^{n+1} d x \geqslant 0.5$. The bubble volume is strictly conserved when using the Variant 4 and decreases when using the Variant 3. This volume decrease is less important when the time step go to zero but is still present. Note that the same kind of behaviour is observed for the quantity $\int_{\Omega} c_{h}^{n+1} d x$. This can be explained by the apparition of spurious velocities in the neighborhood of interfaces (see Figure 6) which brings here a consequent contribution to the volume evolution through the transport term $\int_{\Omega} \mathbf{u}_{h}^{n} \cdot \nabla c_{h}^{n+1} d x$. Indeed, as it was mentioned in the proof of Theorem 1, the transport velocity $\mathbf{u}_{h}^{n}$ is not divergence free, it is the $\mathrm{L}^{2}$ projection on $\mathcal{V}_{h, 0}^{\mathbf{u}, n+1}$ of the function $\widetilde{\mathbf{u}}_{h}^{n+1}-\Delta t \nabla \Phi_{h}^{n+1}$ which is divergence free in a weak sense (i.e. against functions of $\mathcal{V}_{h}^{p, n+1}$ ). This is true for both Variants 3 and 4 but consequences are less serious for Variant 4 thanks to spurious currents elimination. 


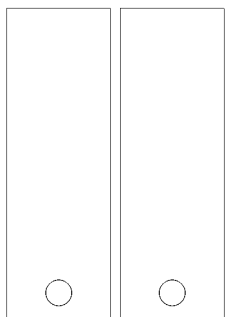

$t=0$

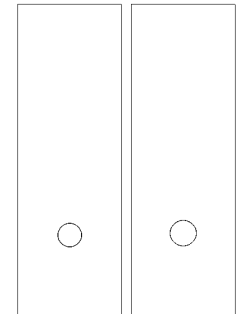

$t=0.14$

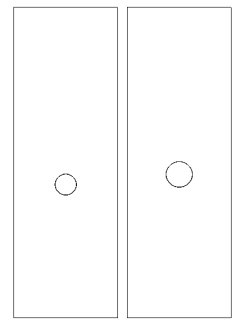

$t=0.28$

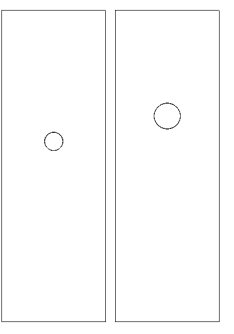

$t=0.42$

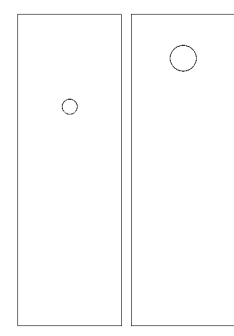

$t=0.56$

Figure 4: Time evolution $\left(\Delta t=5 \times 10^{-4}\right)$ of the system using the Variant 3 (on the left) and the Variant 4 (on the right).

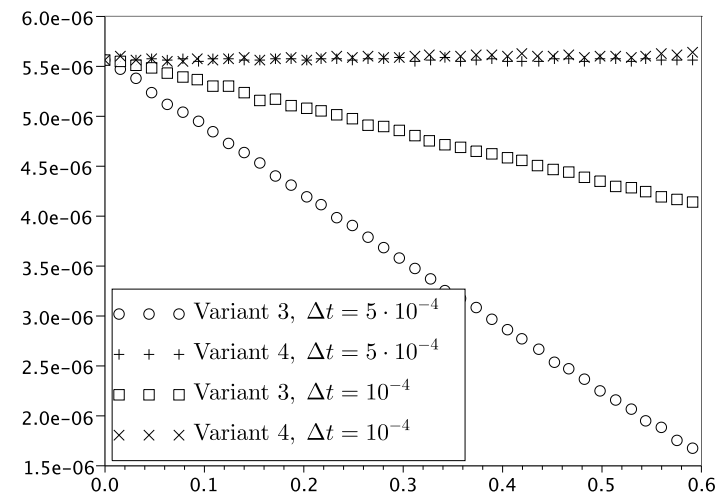

Figure 5: Time evolution of the volume of the bubble. 


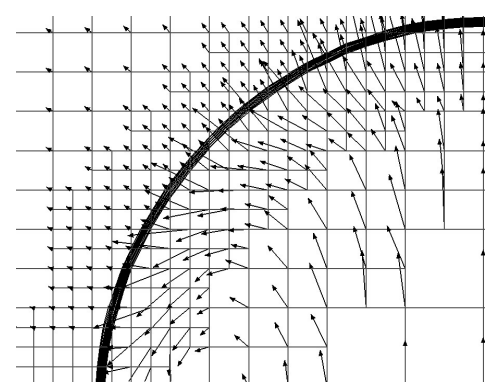

Variant 3

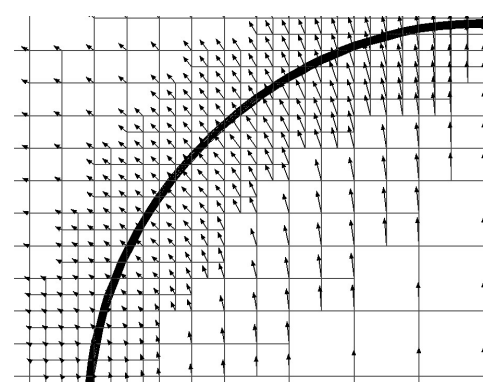

Variant 4

Figure 6: Velocity at $t=0.28$ using the Variant 3 (on the left) and the Variant 4 (on the right).

\section{Conclusion}

In this article, we proposed a new variant ( $c f$ Problem 4) of the incremental projection algorithm. This stable scheme can be used in combination with an adaptive local refinement procedure for the space discretization. Furthermore, it eliminates the spurious velocities due to the taking into account of the right-hand side of the momentum balance. The behaviour of the method was illustrated with two phase flows simulations using a Cahn-Hilliard/Navier-Stokes model.

\section{References}

[1] J.-L. Guermond, P. Minev, J. Shen, An overview of projection methods for incompressible flows, Comput. Methods Appl. Mech. Engrg 195 (2006) 6011-6045.

[2] K. Goda, A multistep technique with implicit difference schemes for calculating two- or three-dimensional cavity flows, Journal of Computational Physics 30 (1979) 76-95.

[3] J.-L. Guermond, P. Minev, J. Shen, Error analysis of pressure-correction schemes for the time-dependent stokes equations with open boundary conditions, SIAM Journal on Numerical Analysis 43 (1) (2005) 239-258.

[4] A. Ern, J.-L. Guermond, Theory and Pratice of Finite Elements, Vol. 159 of Applied Mathmatical Sciences, Springer, 2004.

[5] J.-L. Guermond, L. Quartapelle, On the approximation of the unsteady Navier-Stokes equations by finite element projection methods, Numer. Math. 80 (2) (1998) 207-238. 
[6] J.-L. Guermond, Un résultat de convergence d'ordre deux en temps pour l'approximation des équations de Navier-Stokes par une technique de projection incrémentale, Mathematical Modelling and Numerical Analysis 33 (1) (1999) 169-189.

[7] P. Angot, M. Jobelin, J.-C. Latché, Error analysis of the penalty-projection method for the time dependent Stokes equations, Int. J. Finite Vol. 6 (1) (2009) 26.

[8] S. Ganesan, V. John, Pressure separation - a technique for improving the velocity error in finite element discretisations of the Navier-Stokes equations, Appl. Math. Comput. 165 (2) (2005) 275-290.

[9] S. Turek, A. Ouazzi, J. Hron, On pressure separation algorithms (PSepA) for improving the accuracy of incompressible flow simulations, Internat. J. Numer. Methods Fluids 59 (4) (2009) 387-403.

[10] J.-F. Gerbeau, C. Le Bris, M. Bercovier, Spurious velocities in the steady flow of an incompressible fluid subjected to external forces, Internat. J. Numer. Methods Fluids 25 (6) (1997) 679-695.

[11] D. M. Anderson, G. B. McFadden, A. A. Wheeler, Diffuse-interface methods in fluid mechanics, in: Annual review of fluid mechanics, Vol. 30, Vol. 30 of Annu. Rev. Fluid Mech., Annual Reviews, Palo Alto, CA, 1998, pp. 139165 .

[12] F. Boyer, A theoretical and numerical model for the study of incompressible mixture flows, Comput. Fluids 31 (1) (2002) 41-68.

[13] D. Jacqmin, Calculation of two-phase Navier-Stokes flows using phase-field modeling, J. Comput. Phys. 155 (1) (1999) 96-127.

[14] C. Liu, J. Shen, A phase field model for the mixture of two incompressible fluids and its approximation by a Fourier-spectral method, Phys. D 179 (34) (2003) 211-228.

[15] J.-L. Guermond, L. Quartapelle, A projection FEM for variable density incompressible flows, J. Comput. Phys. 165 (1) (2000) 167-188.

[16] F. Boyer, C. Lapuerta, S. Minjeaud, B. Piar, A local adaptive refinement method with multigrid preconditionning illustrated by multiphase flows simulations, ESAIM Proceedings 27 (2009) 15-53.

[17] T. Bonometti, J. Magnaudet, An interface-capturing method for incompressible two-phase flows. validation and application to bubble dynamics, International Journal of Multiphase Flow 33 (2) (2007) 109-133.

[18] R. Clift, J. R. Grace, M. E. Weber, Bubbles, drops and particles, Academic Press, New York. 